\title{
Control of Development and Transposon Movement by DNA Methylation in Arabidopsis thaliana
}

\author{
T. Kakutani, M. Kato, T. Kinoshita, and A. Miura \\ Department of Integrated Genetics, National Institute of Genetics, Mishima, \\ Shizuoka 411-8540, Japan
}

In both vertebrates and plants, mutations abolishing DNA methylation induce developmental abnormalities, which often accompany perturbation of transcription (for review, see Richards 1997; Habu et al. 2001; Li 2002). A unique feature in plants is that the induced epigenetic changes are often inherited over multiple generations even after introduction into a wild-type background (for review, see Martienssen 1998; Kakutani 2002). This feature has facilitated identification of plant genes controlled by DNA methylation through conventional genetic linkage analysis. Tissue-specific as well as parent-of-origin-specific transcriptional control by DNA methylation can be studied further using Arabidopsis mutants affecting epigenetic states.

Control of transcription of genes involved in development is not the only function of DNA methylation. In vertebrates, plants, and some fungi, methylated cytosine is often found in repeated sequences including transposons. One model to explain this distribution proposes that the primary function of eukaryotic DNA methylation is to protect the genome from the deleterious effects of transposons (Yoder et al. 1997; Matzke et al. 1999; Selker et al. 2003). Consistent with such a "genome defense" hypothesis, endogenous Arabidopsis transposons are mobilized by mutations affecting genomic DNA methylation (Miura et al. 2001; Singer et al. 2001). In this paper, we summarize our genetic approaches to understand the roles of DNA methylation in the control of development and behavior of transposons.

\section{DEVELOPMENTAL ABNORMALITIES INDUCED BY ARABIDOPSIS DNA METHYLATION MUTANTS}

Arabidopsis provides a genetically tractable system to examine the role of DNA methylation, because viable mutants defective in DNA methylation are available. MET1 (METHYLTRANSFERASE1, an ortholog of the mammalian DNA methyltransferase Dnmt1) is necessary for maintaining genomic cytosine methylation at 5'-CG3' sites (Finnegan et al. 1996; Ronemus et al 1996). Arabidopsis additionally methylates non-CG sites using CHROMOMETHYLASE3 (CMT3) (Bartee et al. 2001; Lindroth et al. 2001). A third Arabidopsis gene necessary for DNA methylation is DDMI (DECREASE IN DNA METHYLATION1), which encodes a chromatin remodeling factor (Vongs et al. 1993; Jeddeloh et al. 1999; Brzeski and Jerzmanowski 2002).

A striking feature of the $d d m 1$ DNA hypomethylation mutation is that it results in a variety of developmental abnormalities by inducing heritable changes in other loci (Fig. 1) (Kakutani et al. 1996; Kakutani 1997). Target loci have been identified for three of $d d m 1$-induced heritable developmental abnormalities. Two of them, $F W A$ and $B A L$, are caused by epigenetic changes in transcription, and one of them, CLAM, is due to a genetic change, a transposon insertion. Each of them will be discussed in the following sections.

\section{FWA: INHERITANCE OF IMPRINTING OVER MULTIPLE GENERATIONS}

The $d d m 1$-induced phenotype observed most frequently is delay in flowering initiation (Kakutani et al. 1996; Kakutani 1997). Linkage analysis and molecular analysis revealed that this phenotype was due to ectopic transcription of a homeobox gene $F W A$ (Koornneef et al. 1991; Kakutani 1997; Soppe et al. 2000). The FWA gene is transcriptionally silent in wild-type adult tissues, but the transcript accumulates in the $d d m 1$-induced late-flowering
Figure 1. Developmental abnormalities found in $d d m 1$ DNA hypomethylation mutant lines. Each of the $d d m 1$ lines has been self-pollinated seven times independently. (a) Flowers of a wild-type Col plant. (b) Flowers with thin sepals in a $d d m 1$ line. (c) Homeotic transformation of ovule into other flower organs observed in a $d d m 1$ line. (d) Right, upward leaf curling in a $d d m 1$ line. Left, control wild-type Columbia plant. (e) bonsai phenotype in a $d d m 1$ line.
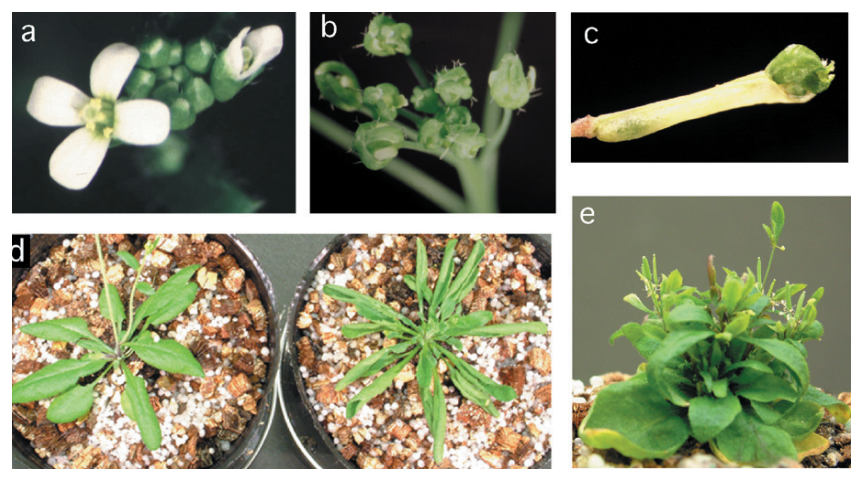
lines (Soppe et al. 2000). Interestingly, the $d d m 1$-induced transcriptional $F W A$ activation and late-flowering phenotypes are heritable over multiple generations, which allowed the identification of $F W A$ locus by the linkage analysis (Kakutani 1997; A. Miura and T. Kakutani, unpubl.). A similar phenotype is also induced by a loss of function in the CG methyltransferase gene MET1, indicating that DNA methylation is critical for the silencing of the FWA gene (Ronemus et al. 1996; Kankel et al. 2003).

Although the $F W A$ transcript is undetectable in wildtype adult tissues, further characterization of the $F W A$ transcription during earlier developmental stages revealed that even in wild-type plants the $F W A$ transcript accumulates in endosperm, a tissue supporting embryo growth in the seed (Fig. 2a) (Kinoshita et al. 2004). In addition, $F W A$ transcription in the endosperm is imprinted; only the maternal-origin transcript was detected (Fig. 2b) (Kinoshita et al. 2004). The silencing of $F W A$ in embryonic tissues and silencing of paternal-origin copy depend on the MET1 gene function (Kinoshita et al 2004). Thus CG methylation is necessary for maintenance of FWA imprinting, as is the case in many of imprinted mammalian genes ( $\mathrm{Li}$ et al 1993). Unlike mammals, however, the maternal-specific $F W A$ expression was not established by paternal-specific de novo methylation; it is established by maternal-specific activation in the central cell of female gametophyte, which depends on the DNA glycosylase gene, DEMETER (Choi et al. 2002; Kinoshita et al. 2004). Thus the silent methylated state is the default for this class of imprinted genes. Resilencing of the activated $F W A$ gene is not necessary, because endosperm does not contribute to the next generation. When methylation is lost in the embryonic lineage (e.g., by $d d m 1$ or met 1 mutation), the fwa epigenetic mutation and the associated late-flowering phenotype can be stably inherited over multiple generations. (a)
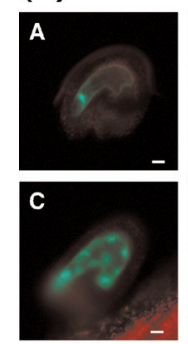
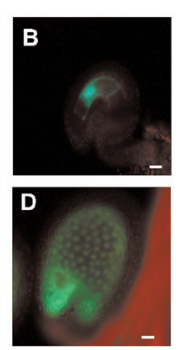

(b)

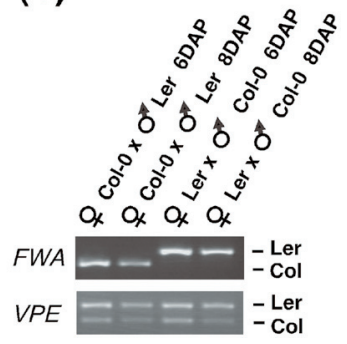

Figure 2. Endosperm-specific and imprinted transcription of $F W A$ gene during early development in wild-type Col plants. (a) Expression pattern of the $F W A$ gene examined by fluorescence images of the $p F W A: \because N L S-G F P$ transgenic plants. (A) Green fluorescent protein (GFP) expression visible in mature central cell nucleus before fertilization. $(B)$ Primary endosperm nucleus after fertilization. (C) 16-32 nuclei endosperm stage. (D) Heart embryo stage corresponding to 5 days after pollination. When the entire $F W A$ coding region was fused to the GFP, the signal disappeared at an earlier stage (Kinoshita et al. 2004). (b) Imprinted transcription of FWA in endosperm. Wild-type Col and Ler strains were used as parents for bidirectional crosses. RTPCR shows maternal-allele-specific expression in the endosperm at 6 and 8 days after pollination (DAP). A VPE gene was used as a control. (Reprinted, with permission, from Kinoshita et al. 2004 [@AAAS].)

\section{OTHER EPIGENETIC ALLELES: bal, sup-LIKE, AND ag-LIKE}

Another $d d m 1$-induced developmental abnormality, bal, maps to a locus with clustered disease resistance genes; and it is also associated with overexpression of sequences within this region (Stokes et al. 2002). Like fwa, this phenotype is stably inherited over multiple generations even after introduction into wild-type $D D M 1$ background (Stokes et al. 2002). Details of the inheritance of bal phenotype are described in another chapter (Yi et al., this volume).

Both bal and fwa phenotypes are due to overexpression induced by the loss of DNA methylation. On the other hand, some of the $d d m 1$-induced phenotypes are due to transcriptional suppression. Floral pattern phenotypes similar to those of superman (sup) or agamous (ag) mutants have been induced in $d d m 1$ or met 1 mutant background. Each of the phenotypes is associated with transcriptional suppression and hypermethylation of the SUP or $A G$ gene (Jacobsen et al. 2000). Interestingly, a global decrease in DNA methylation level in these mutants is associated with local hypermethylation in $S U P$ and $A G$ locus. Although the mechanism is unknown, a similar phenomenon is seen in cancer cells; they can simultaneously show genome-wide hypomethylation and hypermethylation of specific genes (Jacobsen et al. 2000).

\section{OTHER $d d m$ 1-INDUCED DEVELOPMENTAL ABNORMALITIES}

Other types of abnormalities in flower pattern formation have been found in the self-pollinated $d d m 1$ lines, such as decrease in sepal number, thin sepals, and homeotic transformation of ovules into other flower organs (Fig. 1b,c) (Kakutani et al 1996). Upward curling of leaves was also induced in several independent $d d m 1$ lines (Fig. 1d). This phenotype was inherited as a dominant trait over generations in the presence of wild-type $D D M 1$ gene (T. Kakutani, unpubl.). Altered phyllotaxy, clustered siliques, and dwarfism were also found in multiple independent $d d m 1$ lines (Fig. 1e). This combination of phenotypes, which was named bonsai (Kakutani 1997), was inherited as an unstable recessive trait, and it was genetically mapped to the bottom arm of chromosome 1 (T. Kakutani, unpubl.). Target loci for these abnormalities have not been identified yet at the molecular level.

On the other hand, the characterization of another example of heritable $d d m 1$-induced abnormality, clam, lead us to identify a transposon-induced genome rearrangement in DNA hypomethylation backgrounds, as will be described in the next section.

\section{MOBILIZATION OF CACTA TRANSPOSONS BY DNA HYPOMETHYLATION MUTATIONS}

The $d d m 1$-induced clam phenotype was unstable; phenotypically normal sectors were occasionally observed (Fig. 3). Through genetic characterization of the clam phenotype, we identified an endogenous Arabidopsis transposon, named CACTA1. The CACTA1 has features 

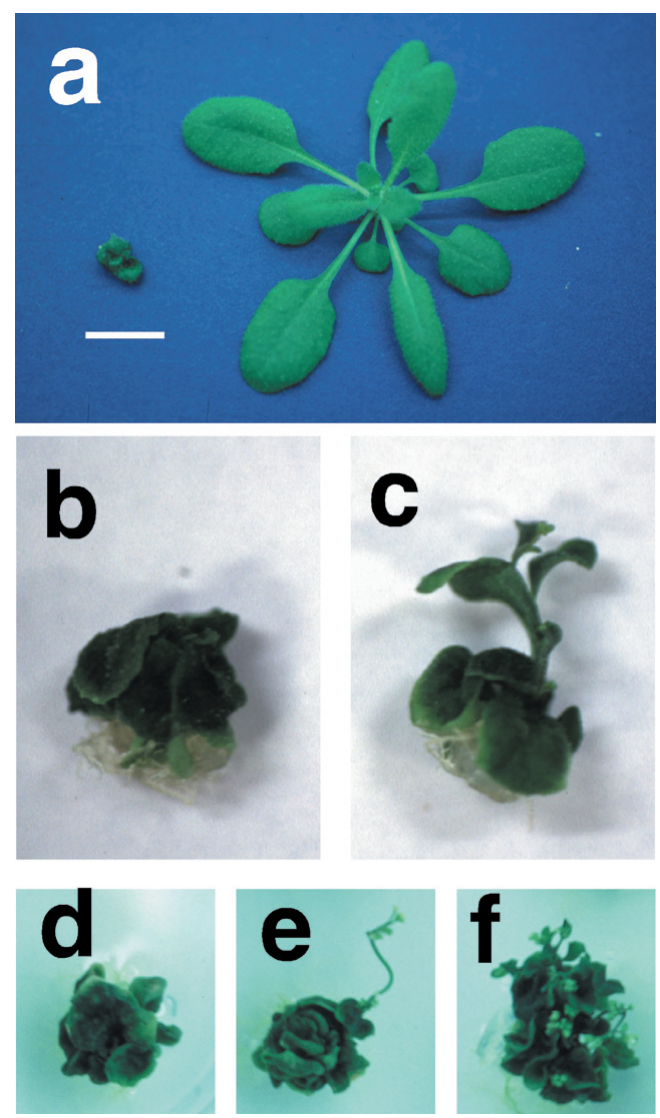

Figure 3. clam phenotype and its reversion. (a) A wild-type Columbia plant showing expanded leaves (right), and a clam homozygous plant with defects in leaf and stem elongation (left). Both are 35-day old. (b-f) The clam homozygous plants with $(c, e, f)$ or without $(b, d)$ reversion sectors. Plants were grown on soil $(a)$ or nutrient agar plates $(b-f)$. White bar: $10 \mathrm{~mm}$. (Reprinted, with permission, from Miura et al. 2001 [@Nature Publishing Group (http://www.nature.com/)].)

typical of the CACTA family DNA-type transposons found in many plant species; the 5' and $3^{\prime}$ ends of which have the conserved terminal inverted sequence CACTACAA. The clam phenotype was due to insertion of a CACTA transposon into DWF4, a gene involved in regulation of plant growth. The phenotypic reversion of clam was associated with excision of the transposon. CACTA1 is silent in wild-type Columbia ( $\mathrm{Col})$ strain, but transposes and increases in the copy number in a $d d m 1$ DNA hypomethylation background (Miura et al. 2001).

The CACTA family transposons are found in many plant species. The CACTA member identified first and studied most extensively is the maize Suppressor-mutator (Spm)/Enhancer (Spm) transposons (for review, see Fedoroff 1996). McClintock found in the 1950s that the activity of Spm transposon changes heritably and reversibly (McClintock 1958). Subsequent molecular characterization of the Spm revealed that changes in transposon activity correlate with epigenetic changes in DNA methylation of element sequences (Fedoroff 1996).

Indeed, loss of DNA methylation seems to be sufficient for mobilization of the Arabidopsis CACTA1, because this element was mobilized in mutants of DNA methyltrans-
Table 1. Transposition of CACTA Elements in ddm1, met1, cmt3, and met1-cmt3 Double Mutants Detected by Changes in the Banding Pattern by Southern Analysis

\begin{tabular}{|c|c|c|c|}
\hline \multirow[b]{2}{*}{$\begin{array}{l}\text { Parental } \\
\text { genotype }\end{array}$} & \multirow[b]{2}{*}{$\begin{array}{c}\mathrm{F}_{2} \\
\text { Genotype }\end{array}$} & \multicolumn{2}{|c|}{$\begin{array}{c}\mathrm{F}_{3}: \text { self-pollinated } \\
\text { progeny from } \mathrm{F}_{2}\end{array}$} \\
\hline & & $\begin{array}{l}\text { Number } \\
\text { examined }^{a}\end{array}$ & $\begin{array}{l}\text { Number of } \\
\text { transposants }\end{array}$ \\
\hline \multirow[t]{2}{*}{$\overline{D D M 1 / d d m 1}$} & $d d m 1 / d d m 1$ & 29 & $17(59 \%)$ \\
\hline & $D D M 1 / D D M 1$ & 35 & 0 \\
\hline \multirow[t]{2}{*}{ MET1/met1 } & met1/met1 & 42 & 0 \\
\hline & MET1/MET1 & 12 & 0 \\
\hline \multirow[t]{2}{*}{ CMT3/cmt3 } & $\mathrm{cmt} 3 / \mathrm{cmt} 3$ & 64 & 0 \\
\hline & СMT3/CMT3 & 48 & 0 \\
\hline MET1/met1- & cmt3-met $1^{\mathrm{c}}$ & 39 & $38(97 \%)$ \\
\hline \multirow[t]{3}{*}{ CMT3/cmt3 } & CMT3-met $1^{\mathrm{c}}$ & 45 & 0 \\
\hline & $c m t 3-M E T 1^{\mathrm{c}}$ & 42 & $4(10 \%)$ \\
\hline & $C M T 3-M E T 1^{\mathrm{c}}$ & 43 & 0 \\
\hline
\end{tabular}

${ }^{\mathrm{a} N u m b e r}$ of $\mathrm{F}_{3}$ plants examined by Southern analysis.

${ }^{b}$ Number of plants with bands in new positions detectable by the Southern analysis.

${ }^{\mathrm{c}}$ Homozygotes for the CMT3 and MET1 loci.

This table summarizes, with permission, the results shown in Table 1 and Supplementary Figure S3 of Kato et al. 2003 (๑) Elsevier)

ferase genes, MET1 (maintenance CG methylase) and CMT3 (non-CG methylase). High-frequency transposition of CACTA elements was detected in cmt3-met1 double mutants. However, single mutants in either met 1 or $\mathrm{cmt} 3$ were much less effective in the mobilization (Table 1) (Kato et al. 2003). Transposition was not detected by Southern analysis in either single mutant segregating from the respective heterozygotes. This observation was confirmed by a polymerase chain reaction (PCR)-based method, in which the original CACTA1 locus with somatic excision less than 1/100 was detectable (Fig. 4).

Interestingly, low frequency of transposition was detected in cmt 3 single mutant homozygotes segregating from self-pollinated progeny of MET1/met1 CMT3/cmt3 double heterozygotes (Table 1) (Kato et al. 2003). This correlates with the accumulation of CACTA1 transcript in a

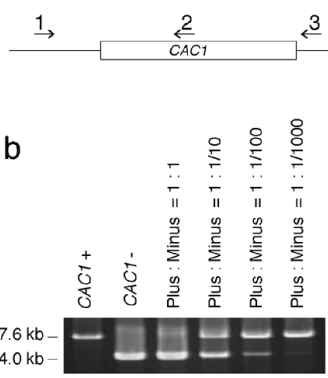

C

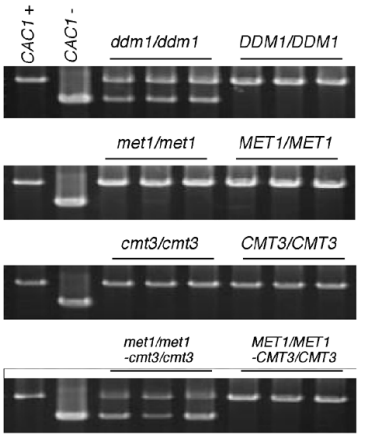

Figure 4. Excision of CACTAl detected by PCR. (a) Schematic drawing of the strategy. Products from primers 1 and 3 and primers 1 and 2 will be amplified from template genomic DNA with or without the excision of CACTA1 from this locus, respectively. (b) Sensitivity of this system. The $4.0-\mathrm{kb}$ product was amplified more efficiently than the 7.6-kb product. (c) Excision of CACTA1 detected in $d d m 1$, met $1, \mathrm{~cm} 3$, or met 1 -cmt 3 mutants segregating in self-pollinated progeny of heterozygotes. Each of the genotypes is compared to wild-type homozygotes in the corresponding segregating families. The primer sequences and PCR conditions will be provided on request. 
these plants, which was undetectable in cmt 3 single mutants segregating in the progeny of $C M T 3 / \mathrm{cmt} 3$ single heterozygotes (not shown). The met 1 mutation, even in heterozygous state, seems to affect the CACTA1 transcription of the progeny. That may be because met $1 \mathrm{mu}-$ tations heritably activate transcription of silent sequences during the haploid gametophyte generation (Kankel et al. 2003; Saze et al. 2003)

The mobilization of CACTA elements may be mediated by changes in chromatin structure, because histone modifications are also affected by the DNA methyltransferase mutants (Johnson et al. 2002; Soppe et al. 2002; Lippman et al. 2003). Although downstream mechanisms remain to be clarified, our results suggest that $\mathrm{CG}$ and non-CG methylation systems redundantly function as heritable epigenetic tags for immobilization of CACTA transposons.

\section{FACTORS DETERMINING TRANSPOSON DISTRIBUTION WITHIN GENOME: INTEGRATION SPECIFICITY VERSUS NATURAL SELECTION}

Transposons are not distributed randomly within genomes. Transposon-related sequences are often enriched in centromeric and pericentromeric regions in many organisms including Arabidopsis (Arabidopsis Genome Initiative 2000). The clustering of the transposons in these heterochromatic regions can be due to preferential integration of transposons into these regions (Boeke and Devine 1998) or can be caused by purifying selection against transposon insertion into gene-rich chromosomal arm regions in natural populations. To evaluate the possible contribution of natural selection, we examined the distribution of the CACTA transposons in genomes of 19 natural Arabidopsis variants (ecotypes) in comparison to new integration sites induced in the laboratory.

Sequences similar to mobile CACTA1 are distributed among the ecotypes and show a strikingly high polymorphism in genomic localization (Miura et al. 2004). This suggests that CACTA1 has transposed in the recent past in natural populations. Despite the high polymorphism, the copy number of CACTA1 was low in all the examined ecotypes (Miura et al. 2004). In addition, they are localized preferentially in pericentromeric and transposonrich regions. This contrasts to transposition induced in laboratory in the $d d m 1$ mutant background, in which the integration sites are less biased and the copy number frequently increases (Miura et al. 2001). The differences in the distribution of integration sites could be due to loss of the heterochromatin marks in the $d d m 1$ mutants, because this mutation affects DNA and histone modification in the heterochromatic regions (Gendrel et al. 2002; Johnson et al. 2002; Soppe et al. 2002). However, when the $C A C T A 1$ element mobilized by $d d m 1$ mutation was introduced into a $D D M 1$ wild-type background, new integration events still were not targeted to the pericentromeric heterochromatin regions (Kato et al. 2004).

Because each of the transposition events does not seem to be biased toward pericentromeric heterochromatin, other factor(s) should be responsible for the accumulation of CACTA1 elements in these regions. One possible mech- anism is that if transposon excision frequency from these heterochromatic regions is low, net accumulation of the "cut-and-paste"-type of transposons in these regions would result. This possibility can be tested experimentally.

Another possible factor is natural selection against deleterious insertion into chromosomal arm regions. This mechanism is consistent with our observation that the copy number of CACTA1 is low in all the examined ecotypes (Miura et al. 2004). If natural selection against deleterious insertions is a significant factor determining transposon distribution, the next question is how the selection operates. An obvious mechanism is that transposon insertions into gene-rich regions sometimes reduce host fitness by direct gene disruption. Other unknown selection mechanisms might also be working, as transposon insertion sometimes have phenotypic effects much stronger than loss of the entire chromosomal region around the insertion site (McClintock 1956).

\section{PERSPECTIVES}

Here we reported that loss of DNA methylation in Arabidopsis induces developmental abnormalities through both transposon mobilization and perturbation of transcription. The change in transcription and associated developmental variation was often heritable over multiple generations. Such an inheritance of differential epigenetic state is an enigmatic phenomenon found often in plants (Jacobsen and Meyerowitz 1997; Cubas et al. 1999; Kakutani et al. 1999; Soppe et al. 2000, 2002; Stokes et al. 2002). Similar inheritance of epigenetic variations has also been reported for some alleles of mammalian genes (Whitelaw and Martin 2001; Rakyan et al. 2003). Interestingly, the affected mammalian alleles often have transposon insertions compared to the wild-type allele. Inheritance of epigenetic silencing may be used, at least for some sequences, for transgenerational genome defense against deleterious genome rearrangement induced by transposon movement. For the genome defense by DNA methylation (Yoder et al. 1997; Matzke et al. 1999), the heritable property might be advantageous, because the silencing is maintained at every stage of development, including early development before the de novo silencing is established (Kato et al 2004).

Components controlling epigenetic states are being clarified in Arabidopsis. In addition, a variety of inbred accessions from natural populations are available, as well as genetic and genomic tools to exploit these resources. Arabidopsis will provide a good system to understand control of transposons, not only of their transcription, but also of their effects on genome integrity and chromosomal evolution.

\section{ACKNOWLEDGMENTS}

Tetsuji Kakutani thanks Eric Richards for advice in the initial stages of the project. Thanks also to Eric Richards and Jun-ichi Tomizawa for critically reading the manuscript. Supported by Grant-in-Aid for Creative Scientific Research 14GS0321 from Japan Society for the Promotion of Science. 


\section{REFERENCES}

Arabidopsis Genome Initiative. 2000. Analysis of the genome sequence of the flowering plant Arabidopsis thaliana. Nature 408: 796.

Bartee L., Malagnac F., and Bender J. 2001. Arabidopsis cmt3 chromomethylase mutations block non-CG methylation and silencing of an endogenous gene. Genes Dev. 15: 1753.

Boeke J.D. and Devine S.E. 1998. Yeast retrotransposons: Finding a nice quiet neighborhood. Cell 93: 1087.

Brzeski J. and Jerzmanowski A. 2002. Deficient in DNA methylation 1 (DDM1) defines a novel family of chromatin remodeling factors. J. Biol. Chem. 278: 823.

Choi Y., Gehring M., Johnson L., Hannon M., Harada J.J., Goldberg R.B., Jacobsen S.E., and Fischer R.L. 2002. DEMETER, a DNA glycosylase domain protein, is required for endosperm gene imprinting and seed viability in Arabidopsis. Cell 110: 33.

Cubas P., Vincent C., and Coen E. 1999. An epigenetic mutation responsible for natural variation in floral symmetry. Nature 401: 157.

Fedoroff N. 1996. Epigenetic regulation of the maize Spm transposable element. In Epigenetic mechanisms of gene regulation (ed. V.E.A. Russo et al.), p 575. Cold Spring Harbor Laboratory Press, Cold Spring Harbor, New York.

Finnegan E., Peacock J., and Dennis E. 1996. Reduced DNA methylation in Arabidopsis thaliana results in abnormal plant development. Proc. Natl. Acad. Sci. 93: 8449.

Gendrel A.V., Lippman Z., Yordan C., Colot V., and Martienssen R.A. 2002. Dependence of heterochromatic histone H3 methylation patterns on the Arabidopsis gene DDM1. Science 297: 1871.

Habu Y., Kakutani T., and Paszkowski J. 2001. Epigenetic developmental mechanisms in plants: Molecules and targets of plant epigenetic regulation. Curr. Opin. Genet. Dev. 11: 215.

Jacobsen S.E. and Meyerowitz E.M. 1997. Hypermethylated SUPERMAN epigenetic alleles in Arabidopsis. Science 277: 1100 .

Jacobsen S.E., Sakai H., Finnegan E.J., Cao X., and Meyerowitz E.M. 2000. Ectopic hypermethylation of flower-specific genes in Arabidopsis. Curr. Biol. 10: 179.

Jeddeloh J.A., Stokes T.L., and Richards E.J. 1999. Maintenance of genomic methylation requires a SWI2/SNF2-like protein. Nat. Genet. 22: 94.

Johnson L., Cao X., and Jacobsen S. 2002. Interplay between two epigenetic marks. DNA methylation and histone H3 lysine 9 methylation. Curr. Biol. 12: 1360.

Kakutani T. 1997. Genetic characterization of late-flowering traits induced by DNA hypomethylation mutation in Arabidopsis thaliana. Plant J. 12: 1447.

. 2002. Epi-alleles in plants: Inheritance of epigenetic information over generations. Plant Cell Physiol. 43: 1106.

Kakutani T., Munakata K., Richards E.J., and Hirochika H. 1999. Meiotically and mitotically stable inheritance of DNA hypomethylation induced by ddm1 mutation of Arabidopsis thaliana. Genetics 151: 831.

Kakutani T., Jeddeloh J., Flowers S., Munakata K., and Richards E. 1996. Developmental abnormalities and epimutations associated with DNA hypomethylation mutations. Proc. Natl. Acad. Sci. 93: 12406.

Kankel M.W., Ramsey D.E., Stokes T.L., Flowers S.K., Haag J.R., Jeddeloh J.A., Riddle N.C., Verbsky M.L., and Richards E.J. 2003. Arabidopsis MET1 cytosine methyltransferase mutants. Genetics 163: 1109.

Kato M., Takashima K., and Kakutani T. 2004. Epigenetic control of CACTA transposon mobility in Arabidopsis thaliana. Genetics 168: 961.

Kato M., Miura A., Bender J., Jacobsen S.E., and Kakutani T. 2003. Role of CG and non-CG methylation in immobilization of transposons in Arabidopsis. Curr. Biol. 13: 421.

Kinoshita T., Miura A., Choi Y., Kinoshita Y., Cao X., Jacobsen S.E., Fischer R., and Kakutani T. 2004. One-way control of FWA imprinting in Arabidopsis endosperm by DNA methylation. Science 303: 521.
Koornneef M., Hanhart C.J., and van der Veen J.H. 1991. A genetic and physiological analysis of late flowering mutants in Arabidopsis thaliana. Mol. Gen. Genet. 229: 57.

Li E. 2002. Chromatin modification and epigenetic reprogramming in mammalian development. Nat. Rev. Genet. 3: 662.

Li E., Beard C., and Jaenisch R. 1993. Role for DNA methylation in genomic imprinting. Nature 366: 362.

Lindroth A.M., Cao X., Jackson J.P., Zilberman D., McCallum C.M., Henikoff S., and Jacobsen S.E. 2001. Requirement of CHROMOMETHYLASE3 for maintenance of $\mathrm{CpXpG}$ methylation. Science 292: 2077.

Lippman Z., May B., Yordan C., Singer T., and Martienssen R. 2003. Distinct mechanisms determine transposon inheritance and methylation via small interfering RNA and histone modification. PLoS Biol. 1: E67.

Martienssen R. 1998. Chromosomal imprinting in plants. Curr. Opin. Genet. Dev. 8: 240.

Matzke M.A., Mette M.F., Aufsatz W., Jakowitsch J., and Matzke A.J. 1999. Host defenses to parasitic sequences and the evolution of epigenetic control mechanisms. Genetica 107: 271.

McClintock B. 1956. Controlling elements and the gene. Cold Spring Harbor Symp. Quant. Biol. 21: 197.

. 1958. The Suppressor-mutator system of control of gene action in maize. Carnegie Inst. Wash. Year Book 57: 415.

Miura A., Kato M., Watanabe K., Kawabe A., Kotani H., and Kakutani T. 2004. Genomic localization of endogenous mobile CACTA family transposons in natural variants of Arabidopsis thaliana. Mol. Gen. Genomics 270: 524.

Miura A., Yonebayashi S., Watanabe K., Toyama T., Shimada H., and Kakutani T. 2001. Mobilization of transposons by a mutation abolishing full DNA methylation in Arabidopsis. Nature 411: 212.

Rakyan V.K., Chong S., Champ M.E., Cuthbert P.C., Morgan H.D., Luu K.V., and Whitelaw E. 2003. Transgenerational inheritance of epigenetic states at the murine Axin $(\mathrm{Fu})$ allele occurs after maternal and paternal transmission. Proc. Natl. Acad. Sci. 100: 2538.

Richards E.J. 1997. DNA methylation and plant development. Trends Genet. 13: 319.

Ronemus M., Galbiati M., Ticknor C., Chen J., and Dellaporta S. 1996. Demethylation-induced developmental pleiotropy in Arabidopsis. Science 273: 654.

Saze H., Scheid O.M., and Paszkowski J. 2003. Maintenance of $\mathrm{CpG}$ methylation is essential for epigenetic inheritance during plant gametogenesis. Nat. Genet. 34: 65.

Selker E., Tountas N., Cross S., Margolin B., Murphy J., Bird A., and Freitag M. 2003. The methylated components of the Neurospora genome. Nature 422: 893.

Singer T., Yordan C., and Martienssen R. A. 2001. Robertson's Mutator transposons in A. thaliana are regulated by the chromatin-remodeling gene Decrease in DNA Methylation (DDM1). Genes Dev. 15: 591.

Soppe W.J., Jacobsen S.E., Alonso-Blanco C., Jackson J.P., Kakutani T., Koornneef M., and Peeters A.J. 2000. The late flowering phenotype of fwa mutants is caused by gain-offunction epigenetic alleles of a homeodomain gene. Mol. Cell 6: 791.

Soppe W., Jasencakova Z., Hauben A., Kakutani T., Meister A., Huang M., Jacobsen S., Schubert I., and Fransz P. 2002. DNA methylation controls histone $\mathrm{H} 3$ lysine 9 methylation and heterochromatin assembly in Arabidopsis. EMBO J. 21: 6549.

Stokes T.L., Kunkel B.N., and Richards E.J. 2002. Epigenetic variation in Arabidopsis disease resistance. Genes Dev. 16: 171.

Vongs A., Kakutani T., Martienssen R.A., and Richards E.J. 1993. Arabidopsis thaliana DNA methylation mutants. Science 260: 1926.

Whitelaw E. and Martin D. I. 2001. Retrotransposons as epigenetic mediators of phenotypic variation in mammals. Nat. Genet. 27: 361.

Yoder J.A., Walsh C.P., and Bestor T.H. 1997. Cytosine methylation and the ecology of intragenomic parasites. Trends Genet. 13: 335. 


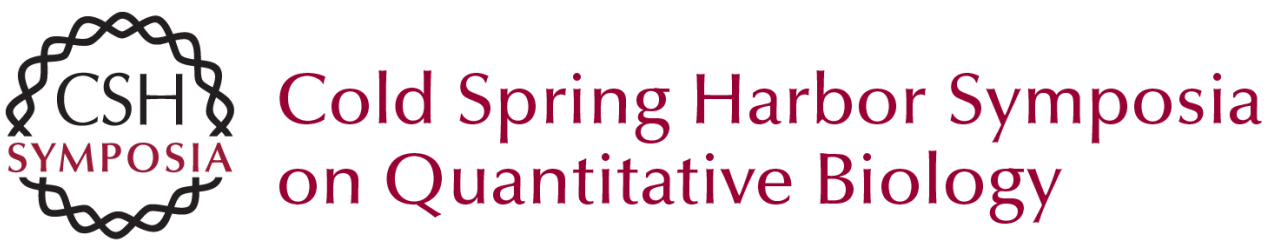

\section{Control of Development and Transposon Movement by DNA Methylation in Arabidopsis thaliana}

T. KAKUTANI, M. KATO, T. KINOSHITA, et al.

Cold Spring Harb Symp Quant Biol 2004 69: 139-144

Access the most recent version at doi:10.1101/sqb.2004.69.139

References This article cites 44 articles, 17 of which can be accessed free at: http://symposium.cshlp.org/content/69/139.full.html\#ref-list-1

License

Email Alerting Receive free email alerts when new articles cite this article - sign up in Service the box at the top right corner of the article or click here. 